\title{
Sex Differences in Multidirectional Neck Muscle Strength Characteristics in High School Soccer Athletes
}

\author{
Takashi Nagai ${ }^{1,2 *}$, Eric M Crowley ${ }^{1}$, Ethan D Manuell ${ }^{2}$, Nathaniel A Bates ${ }^{1,2,3}$, Michael J Stuart ${ }^{1,2}$ and \\ Nathan D Schilaty ${ }^{1,2,3,4}$
}

\author{
${ }^{1}$ Sports Medicine Center, Mayo Clinic, USA \\ ${ }^{2}$ Department of Orthopedic Surgery, Mayo Clinic, USA \\ ${ }^{3}$ Department of Physiology \& Biomedical Engineering, Mayo Clinic, USA \\ ${ }^{4}$ Department of Physical Medicine \& Rehabilitation, Mayo Clinic, USA
}

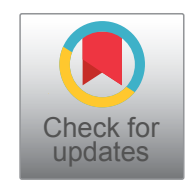

\begin{abstract}
Weaker neck strength has been suggested as a potential risk factor of sport related concussion in female soccer athletes. The purpose of this study was to examine sex differences in neck muscle strength characteristics in multiple directions. A total of 52 high school soccer players (17 Females/35 Males) with no history of concussion participated. Subjects were instructed to perform three maximum voluntary isometric contractions for eight directions: flexion, right / left diagonal flexion, right/left lateral flexion, right/left diagonal extension, and extension. Peak force, normalized peak force, force steadiness, force ratio, and rate of force development were calculated and used for statistical analyses. Based on the normality test on each dependent variable, either independent t-tests or Mann-Whitney U-tests were used to compare sex differences $(P<0.00625$ : Adjusted for multiple comparisons). Males had significantly higher peak force in right lateral flexion and extension directions and rate of force development in left diagonal flexion and left lateral flexion direction compared to females $(P<0.00625)$. After values were normalized to body mass, significant sex differences were only observed with peak force in right lateral flexion. Significant sex differences were found mostly in secondary directions. Continued research efforts are needed to determine how neck strength characteristics may influence sport related concussion risk in soccer athletes.
\end{abstract}

\section{Keywords}

Peak force, Peak force ratio, Force steadiness, Rate of force development, High school soccer athletes, Sex differences, Multidirectional neck strength, Sport related concussion

\section{Introduction}

Sports-related concussion (SRC) is one of the most common injuries among young and athletic individuals with an estimated 1.6 - 3.8 million occurrences each year in the United States [1]. In addition, female athletes have 1.4 times higher rates of SRC compared to male counterparts based on the National Collegiate Athletic Association Injury Surveillance database [2]. This sex discrepancy is in part related to sex differences in reporting behaviors as female athletes are more likely to report occurrences and symptoms of SRC $[3,4]$. Additionally, several potential factors have been identified to explain this sex difference: neck anthropometric parameters, neck muscular strength, types of sport/activities, and a history of SRC [5-8]. Among these factors, neck muscle strength characteristics are modifiable through exercise-based interventions and play an important role in management of SRC in youth athletes [9].

However, the role of neck strength characteristics on neck stiffness regulation and reductions in head acceleration as related to SRC remains unclear. One study reported greater neck strength and anticipatory neck muscle activation (co-contraction) prior to a head perturbation could reduce head displacement from perturbation [10]. Contrarily, neck strength alone did not reduce head impact acceleration in ice hockey players [11]. Additionally, American football players with stronger neck muscles and larger muscle size had higher, odds of sustaining moderate linear head impacts when com-

*Corresponding author: Takashi Nagai, Department of Orthopedic Surgery, Mayo Clinic, Rochester, Minnesota, USA

Accepted: November 10, 2020

Published online: November 12, 2020

Citation: Nagai T, Crowley EM, Manuell ED, et al. (2020) Sex Differences in Multidirectional Neck Muscle Strength Characteristics in High School Soccer Athletes. Arch Sports Med 4(2):224232

Copyright: (c) 2020 Nagai T, et al. This is an open-access article distributed under the terms of the Creative Commons Attribution License, which permits unrestricted use, distribution, and reproduction in any medium, provided the original author and source are credited. 
pared to those players with weaker neck muscles [12]. A recent study with a head/neck musculoskeletal model revealed that ligaments provide more stability than muscles during a football head impact [13]. Similarly, increased cervical muscle force failed to influence head kinematics of a dummy model during simulated concussion events [14].

Clinically, there are few prospective studies to examine a role of neck strength characteristics on SRC occurrences. One study reported that stronger neck muscles could lower concussion risk among high school soccer, basketball, and lacrosse athletes [15]. The authors reported that every pound of increase in neck muscle strength decreased concussion risk by $5 \%$. This study also identified female sex as a risk factor for SRC due largely to their lower neck strength than males [15]. Sex differences in absolute and normalized neck strength (lower in females) have been reported in several studies [5,10,15-17]. As multiple impact locations have been reported as the source of concussion onset in high school soccer athletes [18], evaluation of neck muscle strength and strength ratios in multiple directions are warranted to evaluate cranial stability.

More recently, diminished neck proprioception was reported following SRC, and this deficit was identified as a risk factor of future head/neck injury [19]. It was speculated that severe rotational acceleration head/neck injury could cause damage or dysfunction involving the primary somatosensory cortex and result in altered (longer latency time) somatosensory evoked potentials [20]. A simple force steadiness test (coefficient of variation: CoV of neck force during isometric muscle contractions) was used in the past as a surrogate measurement of the neck sensorimotor characteristics in women with chronic neck pain [21]. We have examined effects of a SRC history on neck force steadiness among high school football players; however, contrary to the hypothesis, no significant differences in $\mathrm{CoV}$ were observed between the players with and without a SRC history [22]. To the authors' knowledge, sex differences in neck force steadiness in high school soccer athletes have not been examined.

As discussed earlier, football players with stronger and larger neck muscles did not mitigate the odds of high magnitude head impact during their football season [12]. Instead, the authors reported that neuromuscular control of neck muscles in anticipation of or reaction to a perturbation/impact might play an important role in reduction of neck angular displacement and increase cervical stiffness and resulted in lower odds of sustaining higher magnitude impacts [12]. Higher rates of force development (RFD) are associated with enhanced neural drive and faster movements as they reflect the rate at which muscle tension can develop. Therefore, it is of interest to continue exploring a role of RFD. To the authors' knowledge, only one study has compared RFD in the neck between males and females in the general population [23]. The authors found that males aged 20-45 had significantly higher RFD in neck flexion and extension directions than age-matched females [23]. Sex differences in multidirectional neck strength and RFD in high school soccer players are largely unknown, and more research is warranted.

The aim of this study was to examine sex differences in neck muscle characteristics among high school soccer players across multiple directions. It was hypothesized that males would produce greater peak force and higher RFD while no sex differences would be observed in peak force ratios and force steadiness. In order to investigate a potential link between weaker neck muscles and SRC in high school soccer athletes, it is clinically relevant for sports medicine specialists and researcher to understand multidirectional neck muscle characteristics in this population.

\section{Materials and Methods}

\section{Participants}

This study was approved by the Mayo Clinic Institutional Review Board. Participants were recruited from three local public high schools at the time of their annual pre-season baseline concussion testing. Only athletes who were cleared by sports medicine physicians to participate in high school athletics were included. Individuals who were not allowed to exert the maximal effort due to neck pain or other neurological/musculoskeletal/medical conditions were excluded from the study. Parent informed written consent and participant's informed assent (if subjects were under 18 years old) was voluntarily obtained prior to data collection. A power analysis was conducted based on the previous study [5], and a number of 14 subjects in each group was defined as the minimum number of subjects needed for this study. A total of 52 high school soccer athletes (35 males, 17 females) participated in this study (age: males $=14.9(1.8)$ years, females $=14.8(1.9)$ years; height: males $=172.9(8.8) \mathrm{cm}$, females $=165.7(8.3)$ $\mathrm{cm}$; and weight: males $=62.7(13.3) \mathrm{kg}$, females $=58.1(11.1)$ $\mathrm{kg})$. Males were significantly taller than females $(P=0.007)$, no other significant differences in demographics were noted between sexes.

\section{Data collection procedures}

All neck muscle characteristics were collected with a custom-built neck strength testing apparatus (Figure 1). Aluminum beams were used to construct a chair frame with a seat and adjustable harnesses. Baseball helmets (Rawlings Coolflo Batting Helmet, Rawlings, St. Louis, MO, USA) with three different sizes (T-ball, Junior, Senior) were bolted to an aluminum plate prior to the experiment. The aluminum plate with a helmet slides under another aluminum plate with a six degree-of-freedom load cell (45E15; JR3, Woodland, CA, USA). The helmet position was adjustable in three linear directions: Anterior-posterior, medial-lateral, and superior-inferior. Shoulder and waist harnesses were used to minimize body movement and core muscle recruitment during the tests. The load cell was connected to a multifunction USB data acquisition device (USB-1608G; Measurement Computing, Norton, MA, USA) and a USB isolator (UHR402; Advantech, Milpitas, CA, USA). A laptop display positioned 1 meter anterior from the subject and provided real-time biofeedback on the direction, magnitude, and duration of the force exerted throughout each trial (Figure 1). All data was sampled at $1 \mathrm{kHz}$. 


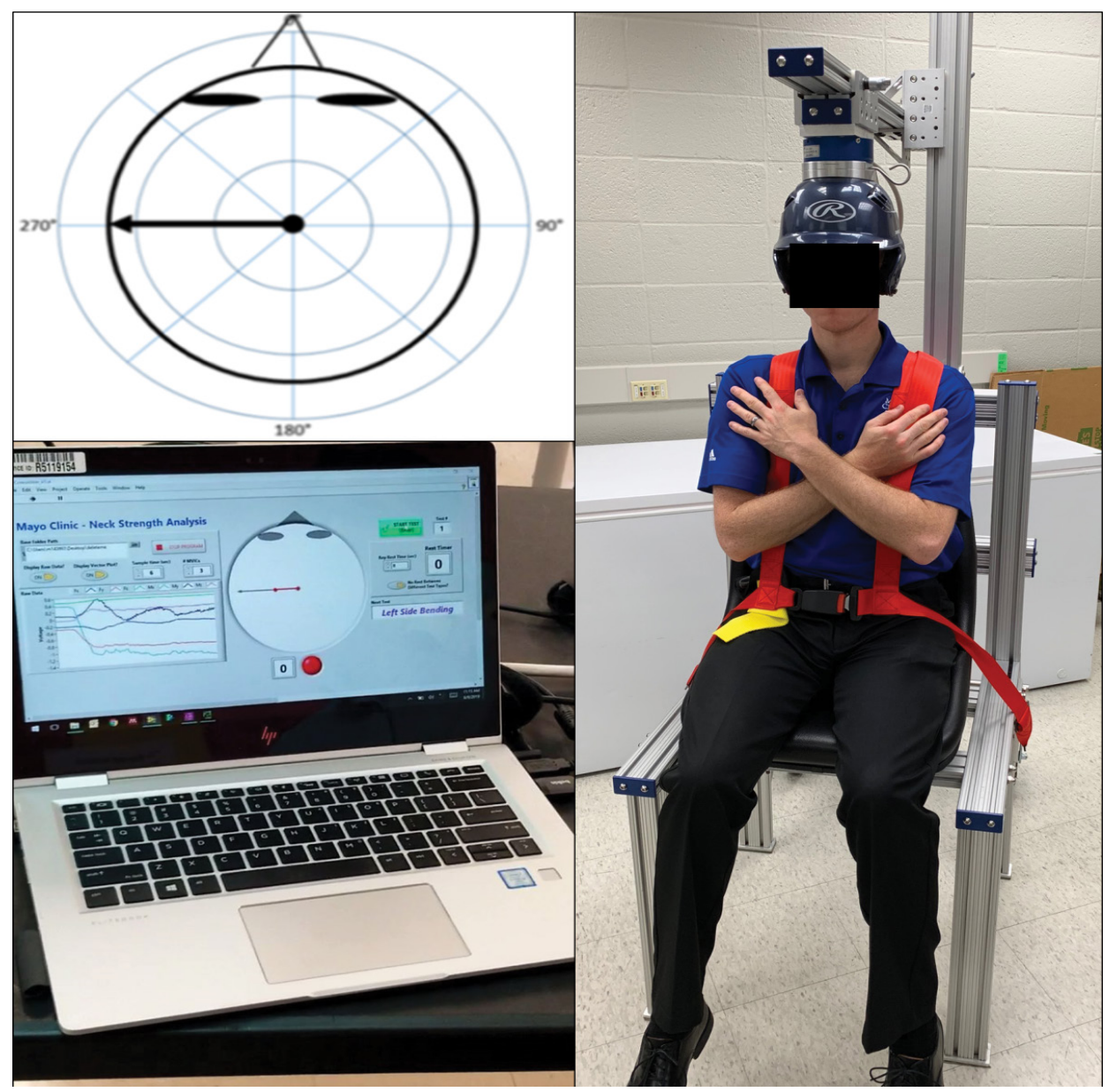

Figure 1: Custom-built neck strength testing apparatus (Right) and screen-shots ( $R=$ Left).

During data collection, participants sat upright in the chair with their head in a neutral position with a helmet. Both the shoulders and waist were fastened with harnesses. A helmet was worn with the front area of the helmet at $1 / 2 \mathrm{~cm}$ above the subject's eyebrows [24]. Participants were instructed to avoid using their trunk muscles, grasping the device with their hands, or pushing against the device with their feet [25]. Verbal instructions and explanations of all testing procedures were provided to the subjects. Three practice trials were provided in each direction prior to the data collection. There were eight different directions (flexion, extension, left/right lateral flexion, left/right $45^{\circ}$ diagonal extension, and left/right $45^{\circ}$ diagonal flexion). A $3 \mathrm{sec}$ maximal voluntary isometric contractions (MVIC) was collected for each trial. Instructions for start/stop and verbal encouragement were provided. A total of three MVIC measurements were performed consecutively for the same direction; then, participants performed the next direction. Subjects were given 45 seconds of rest between contraction sets [25]. Trials were discarded and repeated if subjects did not appropriately push in the guided direction. Order of force direction was randomized.

\section{Data processing}

Absolute peak muscle force was obtained by a one second average of force during the MVIC contraction (within the three second window). Time of instantaneous peak force was used to mark the start of the peak force plateau (Figure 2). For those trials with late instantaneous peak force, time of instantaneous peak force became the final second force plateau. Peak force for statistical analysis was determined by an average of three trials in each direction [11]. Normalized isometric peak force was calculated as absolute isometric peak force divided by subject mass ( $\mathrm{N} / \mathrm{kg}$ ) [11]. The peak force ratios were calculated as absolute isometric peak force for each direction divided by the absolute isometric extension peak force [17]. Conveniently, RFD and peak neck strength can be measured concurrently during MVIC muscle strength testing $[12,26]$. RFD (N/s) was calculated as the change in muscle force ( $\Delta$ force) from onset (at $5 \mathrm{~N}$ ) to $90 \%$ of the peak force divided by change in time ( $\Delta$ time; Figure 2 ). The average of the three trials was used. Normalized RFD (N/s/kg) was calculated as absolute RFD divided by the subject's body weight. Force steadiness was calculated by dividing the standard deviation by the average peak force of the plateau [27]. Test-retest reliability (intraclass coefficient correlation: ICC) for each dependent variable was performed prior to the data collection and found to be moderate to excellent (peak force: ICC = 0.671-0.843; RFD = 0.660- 0.848; CoV: ICC = 0.405-0.698).

\section{Statistical analysis}

Descriptive statistics and independent t-tests were used to examine sex differences. Dependent variables included ab- 
Citation: Nagai T, Crowley EM, Manuell ED, et al. (2020) Sex Differences in Multidirectional Neck Muscle Strength Characteristics in High School Soccer Athletes. Arch Sports Med 4(2):224-232

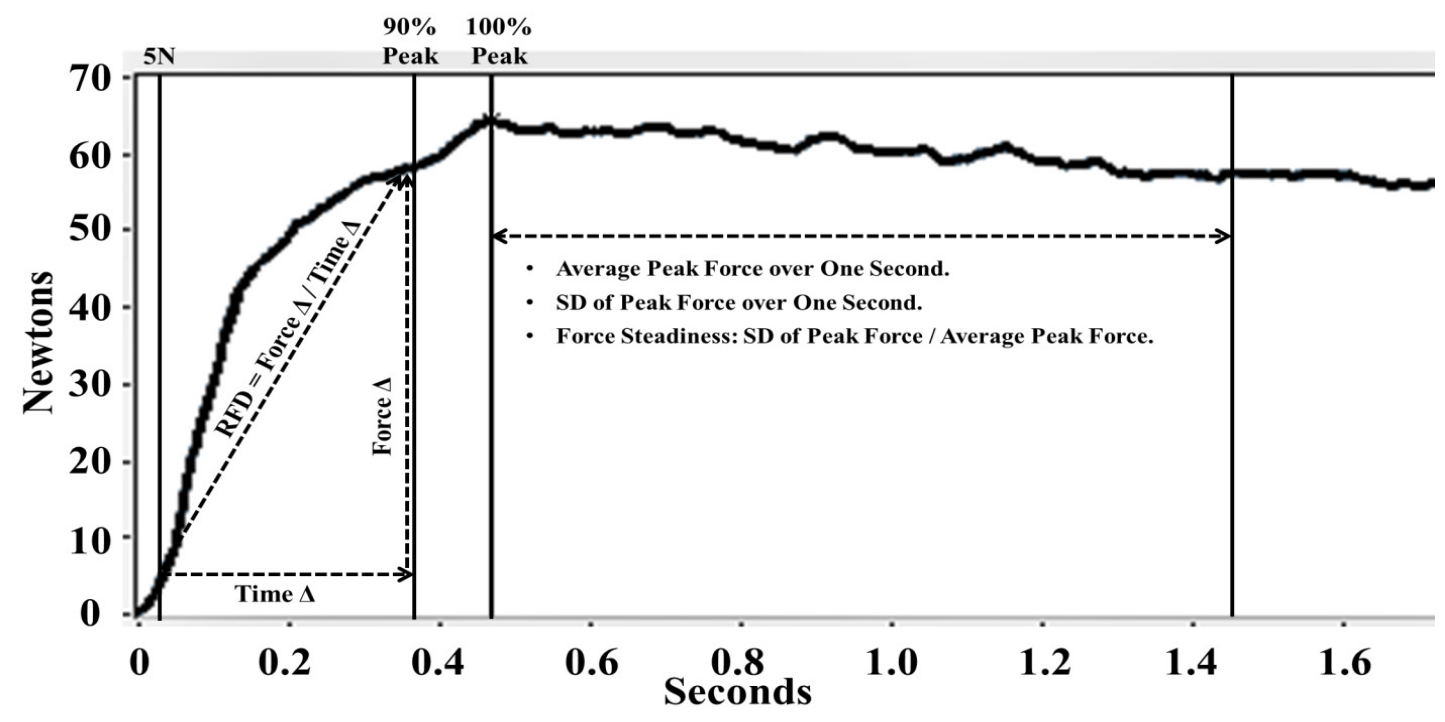

Figure 2: Post-processing and calculations of peak force, force steadiness, and rate of force development (RFD). $\Delta$ : Difference; SD: Standard Deviations.

Table 1: Sex differences in absolute and normalized peak force.

\begin{tabular}{|c|c|c|c|c|c|c|c|}
\hline \multirow[b]{2}{*}{ Absolute Peak Force, $\mathbf{N}$} & \multicolumn{2}{|c|}{ Males $(n=35)$} & \multicolumn{4}{|c|}{ Females $(n=17)$} & \multirow[b]{2}{*}{ Power } \\
\hline & Means & SD & Means & SD & P-Values & Effect Size & \\
\hline \#Flexion & 139.8 & (51.7) & 111.0 & $(27.8)$ & 0.034 & 0.694 & 0.613 \\
\hline \#Right Diagonal Flexion & 118.0 & $(41.2)$ & 100.9 & $(23.3)$ & 0.042 & 0.511 & 0.380 \\
\hline \#Left Diagonal Flexion & 122.4 & $(44.1)$ & 101.3 & $(26.3)$ & 0.079 & 0.581 & 0.469 \\
\hline Right Lateral Flexion & 123.4 & $(35.7)$ & 94.3 & $(20.4)$ & $0.003 *$ & 1.001 & 0.913 \\
\hline \#Left Lateral Flexion & 119.0 & $(38.8)$ & 99.4 & $(19.3)$ & 0.055 & 0.640 & 0.544 \\
\hline Right Diagonal Extension & 143.6 & (37.9) & 120.2 & $(30.8)$ & 0.031 & 0.678 & 0.613 \\
\hline Left Diagonal Extension & 144.3 & (37.1) & 119.3 & $(27.6)$ & 0.017 & 0.765 & 0.718 \\
\hline Extension & 195.5 & $(47.7)$ & 157.6 & $(29.1)$ & $0.004^{*}$ & 0.959 & 0.889 \\
\hline Normalized Peak Force, N/kg & Means & SD & Means & SD & P-Values & Effect Size & Power \\
\hline Flexion & 2.2 & $(0.6)$ & 1.9 & $(0.5)$ & 0.081 & 0.543 & 0.437 \\
\hline Right Diagonal Flexion & 1.9 & $(0.6)$ & 1.8 & $(0.3)$ & 0.225 & 0.167 & 0.086 \\
\hline Left Diagonal Flexion & 2.0 & $(0.5)$ & 1.8 & $(0.4)$ & 0.176 & 0.442 & 0.311 \\
\hline Right Lateral Flexion & 2.0 & $(0.6)$ & 1.6 & $(0.3)$ & $0.005^{*}$ & 0.843 & 0.798 \\
\hline Left Lateral Flexion & 1.9 & $(0.5)$ & 1.7 & $(0.3)$ & 0.113 & 0.485 & 0.363 \\
\hline Right Diagonal Extension & 2.3 & $(0.6)$ & 2.1 & $(0.4)$ & 0.079 & 0.392 & 0.256 \\
\hline Left Diagonal Extension & 2.3 & $(0.5)$ & 2.0 & $(0.4)$ & 0.042 & 0.663 & 0.594 \\
\hline Extension & 3.2 & $(0.8)$ & 2.8 & $(0.6)$ & 0.073 & 0.566 & 0.467 \\
\hline
\end{tabular}

\# represents nonparametric analyses, *represents significant sex differences at $P<0.00625$.

solute peak force, normalized peak force, force ratio, force steadiness, absolute RFD, and normalized RFD in each of eight directions. Each variable was screened for outliers and normality using Shapiro-Wilk tests. Based on normality results, independent t-tests or Mann-Whitney U-tests were performed to compare each variable between sexes. Significance was adjusted for multiple comparisons using Bonferroni and set at $P<0.00625$. All statistical analyses were performed us- ing the statistical software (IBM SPSS v22, IBM Corporation, Armonk, NY, USA).

\section{Results}

Results of descriptive statistics and sex differences are shown in (Table 1, Table 2 and Table 3). All variables on sex differences are summarized in (Figure 3). For absolute peak 
Citation: Nagai T, Crowley EM, Manuell ED, et al. (2020) Sex Differences in Multidirectional Neck Muscle Strength Characteristics in High School Soccer Athletes. Arch Sports Med 4(2):224-232

Table 2: Sex differences in peak force ratios and force steadiness (Coefficient of Variation: CoV).

\begin{tabular}{|c|c|c|c|c|c|c|c|}
\hline \multirow[b]{2}{*}{ Neck Strength Ratio, \% } & \multicolumn{2}{|c|}{ Males $(n=35)$} & \multicolumn{2}{|c|}{ Females $(n=17)$} & \multirow[b]{2}{*}{ P-Values } & \multirow[b]{2}{*}{ Effect size } & \multirow[b]{2}{*}{ Power } \\
\hline & Means & SD & Means & SD & & & \\
\hline Flexion/Extension & 71.4 & $(18.5)$ & 71.5 & $(17.5)$ & 0.984 & 0.006 & 0.050 \\
\hline \#Right Diagonal Flexion/Extension & 60.9 & $(16.0)$ & 64.8 & $(13.8)$ & 0.215 & 0.261 & 0.135 \\
\hline \#Left Diagonal Flexion/Extension & 63.1 & (16.9) & 64.7 & $(13.5)$ & 0.552 & 0.105 & 0.063 \\
\hline Right Lateral Flexion/Extension & 64.7 & $(17.4)$ & 60.2 & (9.8) & 0.239 & 0.319 & 0.185 \\
\hline \#Left Lateral Flexion/Extension & 62.2 & $(18.7)$ & 64.0 & $(11.5)$ & 0.344 & 0.116 & 0.066 \\
\hline Right Diagonal Extension/Extension & 74.3 & $(13.6)$ & 76.1 & $(13.5)$ & 0.659 & 0.133 & 0.073 \\
\hline \#Left Diagonal Extension/Extension & 75.0 & $(15.0)$ & 76.3 & $(15.2)$ & 0.668 & 0.086 & 0.059 \\
\hline Coefficient of Variation, \% & Means & SD & Means & SD & P-Values & Effect Size & Power \\
\hline \#Flexion & 3.7 & (1.6) & 3.1 & (1.0) & 0.501 & 0.449 & 0.308 \\
\hline \#Right Diagonal Flexion & 4.7 & $(2.5)$ & 3.5 & $(1.2)$ & 0.061 & 0.612 & 0.509 \\
\hline \#Left Diagonal Flexion & 4.1 & $(2.0)$ & 3.2 & $(1.2)$ & 0.114 & 0.546 & 0.424 \\
\hline \#Right Lateral Flexion & 4.0 & $(1.7)$ & 3.1 & $(0.8)$ & 0.181 & 0.677 & 0.593 \\
\hline \#Left Lateral Flexion & 4.7 & (3.7) & 3.1 & $(1.2)$ & 0.044 & 0.582 & 0.469 \\
\hline \#Right Diagonal Extension & 4.0 & $(2.2)$ & 3.0 & $(1.6)$ & 0.143 & 0.520 & 0.391 \\
\hline \#Left Diagonal Extension & 3.9 & $(1.7)$ & 2.9 & $(1.2)$ & 0.032 & 0.748 & 0.678 \\
\hline \#Extension & 3.9 & (2.1) & 3.6 & (2.5) & 0.391 & 0.129 & 0.071 \\
\hline
\end{tabular}

${ }^{\#}$ represents nonparametric analyses.

Table 3: Sex differences in absolute and normalized rate of force development (RFD).

\begin{tabular}{|c|c|c|c|c|c|c|c|}
\hline \multirow[b]{2}{*}{ Absolute RFD, N/s } & \multicolumn{4}{|c|}{ Males $(n=35)$} & \multicolumn{3}{|c|}{ Females $(n=17)$} \\
\hline & Means & SD & Means & SD & P-Values & Effect Size & Power \\
\hline Flexion & 194.1 & (183.1) & 111.1 & $(38.4)$ & 0.038 & 0.627 & 0.548 \\
\hline \#Right Diagonal Flexion & 143.4 & $(81.5)$ & 99.7 & $(43.6)$ & 0.071 & 0.669 & 0.581 \\
\hline Left Diagonal Flexion & 156.2 & $(89.3)$ & 100.1 & $(49.4)$ & $0.005^{*}$ & 0.777 & 0.732 \\
\hline \#Right Lateral Flexion & 139.2 & $(87.5)$ & 107.6 & $(48.5)$ & 0.270 & 0.447 & 0.304 \\
\hline \#Left Lateral Flexion & 135.0 & $(73.8)$ & 82.6 & $(36.5)$ & $0.006^{*}$ & 0.901 & 0.830 \\
\hline \#Right Diagonal Extension & 181.4 & $(95.1)$ & 118.5 & $(57.1)$ & 0.014 & 0.802 & 0.738 \\
\hline \#Left Diagonal Extension & 187.1 & $(114.5)$ & 117.6 & $(58.9)$ & 0.023 & 0.763 & 0.696 \\
\hline \#Extension & 278.3 & $(189.3)$ & 182.7 & $(109.2)$ & 0.027 & 0.619 & 0.517 \\
\hline Normalized RFD, N/s/kg & Means & SD & Means & SD & P-Values & Effect Size & Power \\
\hline Flexion & 3.4 & (3.9) & 2.0 & $(0.9)$ & 0.108 & 0.495 & 0.375 \\
\hline \#Right Diagonal Flexion & 2.4 & (1.4) & 1.8 & $(0.8)$ & 0.188 & 0.526 & 0.399 \\
\hline \#Left Diagonal Flexion & 2.7 & (1.9) & 1.7 & $(0.7)$ & 0.081 & 0.698 & 0.619 \\
\hline \#Right Lateral Flexion & 2.3 & (1.4) & 1.9 & $(0.8)$ & 0.592 & 0.351 & 0.206 \\
\hline \#Left Lateral Flexion & 2.2 & $(1.3)$ & 1.4 & $(0.6)$ & 0.023 & 0.790 & 0.725 \\
\hline \#Right Diagonal Extension & 2.9 & (1.5) & 2.0 & $(0.8)$ & 0.022 & 0.749 & 0.679 \\
\hline \#Left Diagonal Extension & 3.1 & $(2.1)$ & 2.0 & $(1.0)$ & 0.084 & 0.669 & 0.581 \\
\hline \#Extension & 4.6 & (3.6) & 3.1 & (1.6) & 0.074 & 0.538 & 0.415 \\
\hline
\end{tabular}

${ }^{\#}$ represents nonparametric analyses. ${ }^{*}$ represents significant sex differences at $P<0.00625$.

forces, males were significantly stronger than females in two directions (right lateral flexion: Males: 123.4 (35.7) N, females: 94.3 (20.4) N, $P=0.003$; extension: Males: 195.5 (47.7) $\mathrm{N}$, females: $157.6(29.1) \mathrm{N}, P=0.004)$. Males were significant- ly stronger in right lateral flexion when normalized peak force was used to compare sex differences (males: 2.0 (0.5) kg, females: $1.6(0.3) \mathrm{kg}, P=0.005)$. No sex differences were observed for neck force ratio and $\operatorname{CoV}(P>0.00625$; Table 2$)$. For 


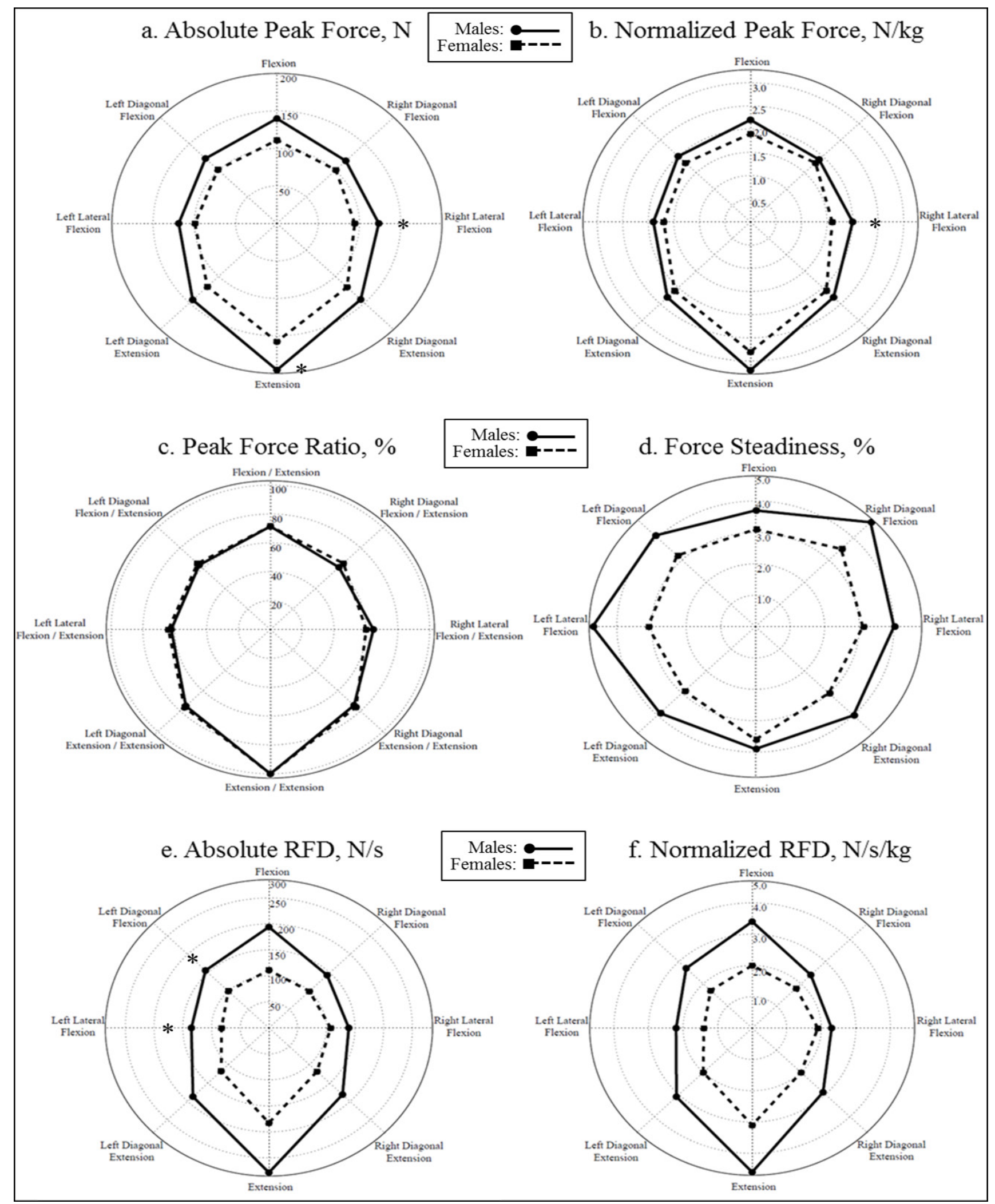

Figure 3: Polar plots of neck muscular force characteristics between sexes. Bold and dotted lines represent males and females, respectively.

*represents $P<0.00625$. RFD: Rate of force development.

absolute RFD, males scored significantly higher than females in left diagonal flexion (males: 156.2 (89.3) N/s, females: $100.1(49.4) \mathrm{N} / \mathrm{s}, P=0.005)$ and left lateral flexion (males:
$135.0(73.8) \mathrm{N} / \mathrm{s}$, females: $82.6(36.5) \mathrm{N} / \mathrm{s}, P=0.006)$. There were no sex differences in normalized RFD $(P>0.00625)$. 


\section{Discussion}

The current investigation aimed to examine sex differences in multidirectional neck muscle characteristics in high school soccer players. For absolute peak force, hypotheses were partially supported as males had significantly higher peak force than females in two directions (right lateral flexion and extension). These results were in agreement with previous studies on sex differences of neck strength $[5,10,15,17]$. Observed peak force in the current investigation were similar to values reported in a previous study with a similar custom-built neck strength apparatus with a 6 degree-of-freedom load cell [28].

\section{Peak force}

Significant neck strength discrepancy in females was still observed in right lateral flexion when the peak force values were normalized to subject's body mass. These results were consistent with a previous study that reported sex differences in absolute and normalized peak force in flexion, extension and lateral flexion directions [17]. Of particular interest, the largest magnitude of sex difference was found in right lateral flexion direction (males had $30.9 \%$ and $25 \%$ higher peak and normalized peak force values than females). Large and superficial lateral flexors muscles (scalene and splenius) and anterior/posterior muscles (sternocleidomastoid/upper trapezius) in the ipsilateral side must work together to generate high lateral flexion peak force [29]. This synchronized muscle activation pattern used during lateral flexion or diagonal flexion tests is more difficult to achieve than flexion and extension tests [30]. It is important to mention that a recent meta-analysis revealed that most concussions occurred because of contact with the ball (heading) or equipment among female soccer athletes [31]. When combined with the current findings (weaker neck strength in lateral directions), baseline neck strength testing might be able to identify female soccer athletes with weak neck strength and monitor future risk of ball-contact related SRC.

\section{Peak force ratio}

Hildenbrand and Vasavada [17] examined neck strength of high school athletes and reported similar peak force ratio values $(0.71$ for flexion/ extension, 0.62 for left lateral flexion/extension, and 0.59 for right lateral flexion/extension) to our current results $(0.71,0.63$, and 0.62 respectively). Further agreement was found in that there were no significant sex differences in neck peak force ratios in either study [17]. However, Kumar and colleagues [32] reported that males had significantly higher peak force ratio for flexion/extension (0.72) than females (0.57). Potential reasons for mixed results are likely related to the differences in demographics of study participants and differences in neck strength measurement instrumentation and procedures. College male athletes generated much higher neck peak forces and higher ratios than high school male athletes while college female athletes did not have as much higher peak force and ratio than high school female athletes [17]. Specific to a heading task in soccer, individuals with greater strength difference between flexion and extension strength (lower flexion/extension ratio) were associated with higher head acceleration during heading while neck strength alone was not associated with head acceleration [33]. Their study did not measure neck strength in the lateral directions; however, based on the current results, neck strength in lateral flexion directions and ratios with neck extension strength were even less than the primary directions and ratio. As a potential solution, a recent study has examined effects of neck strength training on head acceleration; however, the authors did not find significant changes in head acceleration during a heading task [34]. Targeted neck training on neck flexors and lateral flexors to reduce muscle imbalances should be investigated.

\section{Force steadiness}

Few studies have examined sex differences in neck muscle force steadiness. The current results revealed no sex differences; however, there was a trend of higher neck strength CoV. A recent systematic review concluded that females exhibited higher CoV in various extremity muscles [35]. Although there were no sex differences in force steadiness, it was interesting to note that the higher CoV values were observed in secondary directions (right diagonal flexion and left lateral flexion). As discussed earlier, the cervical muscles that were responsible for the secondary neck movements were more difficult to control than the primary movement (flexion and extension). A current study used simple force steadiness as a surrogate measurement of individual's sensorimotor function. Based on our observation from the current study with high school soccer players and another investigation with high school American football players [22], force steadiness using CoV might not be a sensitive measurement. Other measurements such as balance, gait, oculomotor function, and vestibular function were reviewed and demonstrated significant changes in patients with mild traumatic brain injuries [36]. Future investigations should incorporate these measures as a part of comprehensive SRC baseline assessment battery.

\section{Rate of force development}

Another factor worth investigating is RFD. In the current investigation, males produced a $29.4-74.7 \%$ higher absolute RFD than females. Similarly, Valkeinen and colleagues [23] reported that males had $27.3 \%$ and $100 \%$ higher RFD than females in the extension and flexion directions, respectively. For normalized RFD in the current investigation the difference between the sexes was slightly reduced from $29.4-74.7 \%$ to 21.1-70.0\%. The ability to produce force at a faster rate could enable the athlete to react to the external impact force and potentially reduce head impact acceleration [12]. Specific to soccer, female soccer players demonstrated greater head acceleration than male players when they performed a heading task with the ball at the same speed [37]. For soccer players, higher neck strength was associated lower linear and rotational head acceleration during a heading task in soccer [38]. Future studies on RFD between sexes in relation to concussion risk would be needed to establish possibilities for concussion prevention.

\section{Limitations}

It is a limitation that we did not collect some details of the 
Citation: Nagai T, Crowley EM, Manuell ED, et al. (2020) Sex Differences in Multidirectional Neck Muscle Strength Characteristics in High School Soccer Athletes. Arch Sports Med 4(2):224-232

players' profiles - years played, position, or competition level - as these might influence outcomes. Defenders would likely use a heading task more frequently than other positions. Cumulative effects of playing soccer and heading on brain health were discussed elsewhere $[39,40]$. Advances in mobile technology would allow us to capture a player's head acceleration while playing soccer throughout the season. Playing soccer competitively for many years would likely provide sufficient stimulus for an athlete's neck to strengthen over time. If soccer athletes also play other sports, such as wrestling or American football, it is anticipated that they might have higher neck strength as those players usually train neck muscles as a part of their conditioning workout.

\section{Conclusion}

In conclusion, the current investigation revealed that female high school soccer players had less neck muscular peak force and RFD than their male counterparts, as hypothesized. However, after normalization, peak force in right lateral flexion direction was the only significant sex difference found in the study. Importance of balanced neck muscle strength in soccer athletes was discussed.

\section{Acknowledgement}

We acknowledge funding provided by the National Institute of Arthritis and Musculoskeletal and Skin Diseases R01-AR055563 and L30-AR070273, the National Institute of Children and Human Development K12-HD065987, and Mayo Clinic Ultrasound Research Center Grant. The authors would also like to thank Mayo Clinic Sports Medicine Center athletic trainers who conducted baseline concussion testing and helped us to recruit study participants at local high schools: Chad A Eickhoff, Daniel J Christoffer, Kaylan Q Groen, and Mackenzie M Reed.

\section{References}

1. Langlois JA, Rutland-Brown W, Wald M M (2006) The epidemiology and impact of traumatic brain injury: A brief overview. $J$ Head Trauma Rehabil 21: 375-378.

2. Covassin T, Moran R, Elbin RJ (2016) Sex differences in reported concussion injury rates and time loss from participation: An update of the national collegiate athletic association injury surveillance program from 2004-2005 through 2008-2009. J Athl Train 51: 189-194.

3. Wallace J, Covassin T, Beidler E (2017) Sex differences in high school athletes' knowledge of sport-related concussion symptoms and reporting behaviors. J Athl Train 52: 682-688.

4. Kroshus E, Baugh C M, Stein CJ, et al. (2017) Concussion reporting, sex, and conformity to traditional gender norms in young adults. J Adolesc 54: 110-119.

5. Vasavada AN, Danaraj J, Siegmund GP (2008) Head and neck anthropometry, vertebral geometry and neck strength in heightmatched men and women. J Biomech 41: 114-121.

6. Van Pelt KL, Allred D, Cameron KL, et al. (2019) A cohort study to identify and evaluate concussion risk factors across multiple injury settings: Findings from the care consortium. Inj Epidemiol 6: 1.

7. Abrahams S, Fie SM, Patricios J, et al. (2014) Risk factors for sports concussion: An evidence-based systematic review. $\mathrm{Br} \mathrm{J}$ Sports Med 48: 91-97.

8. Pfister T, Pfister K, Hagel B, et al. (2016) The incidence of concussion in youth sports: A systematic review and meta-analysis. $\mathrm{Br} \mathrm{J}$ Sports Med 50: 292-297.

9. Honda J, Chang S H, Kim K (2018) The effects of vision training, neck musculature strength, and reaction time on concussions in an athletic population. J Exerc Rehabil 14: 706-712.

10. Eckner JT, Oh YK, Joshi MS, et al. (2014) Effect of neck muscle strength and anticipatory cervical muscle activation on the kinematic response of the head to impulsive loads. Am J Sports Med 42: 566-576.

11. Mihalik JP, Guskiewicz KM, Marshall SW, et al. (2011) Does cervical muscle strength in youth ice hockey players affect head impact biomechanics? Clin J Sport Med 21: 416-421.

12. Schmidt J D, Guskiewicz K M, Blackburn J T, et al. (2014) The influence of cervical muscle characteristics on head impact biomechanics in football. Am J Sports Med 42: 2056-2066.

13. Kuo C, Sheffels J, Fanton M, et al. (2019) Passive cervical spine ligaments provide stability during head impacts. JR Soc Interface 16: 20190086.

14. Eckersley CP, Nightingale RW, Luck J F, et al. (2019) The role of cervical muscles in mitigating concussion. J Sci Med Sport 22: 667-671.

15. Collins CL, Fletcher EN, Fields SK, et al. (2014) Neck strength: A protective factor reducing risk for concussion in high school sports. J Prim Prev 35: 309-319.

16. Tierney RT, Sitler MR, Swanik CB, et al. (2005) Gender differences in head-neck segment dynamic stabilization during head acceleration. Med Sci Sports Exerc 37: 272-279.

17. Hildenbrand KJ, Vasavada AN (2013) Collegiate and high school athlete neck strength in neutral and rotated postures. J Strength Cond Res 27: 3173-3182.

18. Kerr ZY, Campbell KR, Fraser MA, et al. (2019) Head impact locations in U S High school boys' and girls' soccer concussions, 2012/13-2015/16. J Neurotrauma 36: 2073-2082.

19. Hides JA, Franettovich Smith MM, Mendis MD, et al. (2017) Self-reported concussion history and sensor motor tests predict head/neck injuries. Med Sci Sports Exerc 49: 2385-2393.

20. Zumsteg D, Wennberg R, Gutling E, et al. (2006) Whiplash and concussion: Similar acute changes in middle-latency seps. Can J Neurol Sci 33: 379-386.

21. Muceli S, Farina D, Kirkesola G, et al. (2011) Reduced force steadiness in women with neck pain and the effect of short term vibration. J Electromyogr Kinesiol 21: 283-290.

22. Nagai T, Ueno R, Rigamonti L, et al. (2020) Multidirectional neck strength characteristics in high school football athletes with and without history of sports-related concussion. Great Lakes Athletic Trainers' Association 52nd Annual Meeting and Symposium. Wheeling, IL.

23. Valkeinen H, Ylinen J, Malkia E, et al. (2002) Maximal force, force/time and activation/co activation characteristics of the neck muscles in extension and flexion in healthy men and women at different ages. Eur J Appl Physiol 88: 247-254.

24. Chiu T T, Lam T H, Hedley A J (2002) Maximal isometric muscle strength of the cervical spine in healthy volunteers. Clin Rehabil 16: 772-779. 
Citation: Nagai T, Crowley EM, Manuell ED, et al. (2020) Sex Differences in Multidirectional Neck Muscle Strength Characteristics in High School Soccer Athletes. Arch Sports Med 4(2):224-232

25. Alsalaheen B, Bean R, Almeida A, et al. (2018) Characterization of cervical neuromuscular response to head-neck perturbation in active young adults. J Electromyogr Kinesiol 39: 70-76.

26. Almosnino S, Pelland L, Stevenson JM (2010) Retest reliability of force-time variables of neck muscles under isometric conditions. J Athl Train 45: 453-458.

27. Goetschius J, Hart JM (2016) Knee-extension torque variability and subjective knee function in patients with a history of anterior cruciate ligament reconstruction. J Athl Train 51: 22-27.

28. Gabriel DA, Matsumoto JY, Davis DH, et al. (2004) Multidirectional neck strength and electromyography activity for normal controls. Clin Biomech (Bristol, Avon) 19: 653-658.

29. Kumar S, Narayan Y, Amell T, et al. (2002) Electromyography of superficial cervical muscles with exertion in the sagittal, coronal and oblique planes. Eur Spine J 11: 27-37.

30. Keshner EA, Campbell D, Katz R T, et al. (1989) Neck muscle activation patterns in humans during isometric head stabilization. Exp Brain Res 75: 335-344.

31. Ling D I, Cheng J, Santiago K, et al. (2019) Women are at higher risk for concussions due to ball or equipment contact in soccer and lacrosse. Clin Orthop Relat Res.

32. Kumar S, Narayan Y, Amell T (2001) Cervical strength of young adults in sagittal, coronal, and intermediate planes. Clin Biomech (Bristol, Avon) 16: 380-388.

33. Dezman ZD, Ledet EH, Kerr HA (2013) Neck strength imbalance correlates with increased head acceleration in soccer heading. Sports Health 5: 320-326.

34. Becker S, Berger J, Backfisch M, et al. (2019) Effects of a 6-week strength training of the neck flexors and extensors on the head acceleration during headers in soccer. J Sports Sci Med 18: 729737.

35. Jakobi JM, Haynes EMK, Smart RR (2018) Is there sufficient evidence to explain the cause of sexually dimorphic behaviour in force steadiness? Appl Physiol Nutr Metab 43: 1207-1214.

36. Galea OA, Cottrell MA, Treleaven JM, et al. (2018) Sensorimotor and physiological indicators of impairment in mild traumatic brain injury: A meta-analysis. Neurorehabil Neural Repair 32: $115-128$.

37. Tierney RT, Higgins M, Caswell SV, et al. (2008) Sex differences in head acceleration during heading while wearing soccer headgear. J Athl Train 43: 578-584.

38. Peek K, Elliott J M, Orr R (2019) Higher neck strength is associated with lower head acceleration during purposeful heading in soccer: A systematic review. J Sci Med Sport

39. Kontos A P, Braithwaite R, Chrisman S P D, et al. (2017) Systematic review and meta-analysis of the effects of football heading. Br J Sports Med 51: 1118-1124.

40. Tarnutzer A A, Straumann D, Brugger $P$, et al. (2017) Persistent effects of playing football and associated (sub concussive) head trauma on brain structure and function: A systematic review of the literature. Br J Sports Med 51: 1592-1604.

DOI: $10.36959 / 987 / 257$

Copyright: (c) 2020 Nagai T, et al. This is an open-access article distributed under the terms of the Creative Commons Attribution License, which permits unrestricted use, distribution, and reproduction in any medium, provided the original author and source are credited. 